\title{
[j micromeritics
}

The Science and Technology of Small Particles"

\section{MOF / Metal Organic Frameworks Bibliography}

- Link to press release: Micromeritics Grants an ASAP 2050 Xtended Pressure to Southern Illinois for research that includes MOF.

\begin{tabular}{|c|c|c|c|}
\hline TITLE & AUTHORS & PUBLICATION & AUTHORS' AFFILIATIONS \\
\hline $\begin{array}{l}\text { Copper-based metal- } \\
\text { organic framework for the } \\
\text { facile ring-opening of } \\
\text { epoxides }\end{array}$ & $\begin{array}{l}\text { Jiang, D. I } \\
\text { Mallat, T. I } \\
\text { Krumeich, F. I } \\
\text { Baiker, A. }\end{array}$ & $\begin{array}{l}\text { Journal of Catalysis, } \\
257 \text { (2), p.390-395, } \\
\text { Jul } 2008\end{array}$ & $\begin{array}{l}\text { Department of Chemistry and Applied } \\
\text { Biosciences, ETH Zürich, Hönggerberg, } \\
\text { HCl, } 8093 \text { Zurich , Switzerland }\end{array}$ \\
\hline $\begin{array}{l}\text { Equilibrium, kinetics and } \\
\text { enthalpy of hydrogen } \\
\text { adsorption in MOF-177 }\end{array}$ & \begin{tabular}{|l} 
Saha, D. / Wei, \\
Z. / Deng, S.
\end{tabular} & $\begin{array}{l}\text { International Journal } \\
\text { of Hydrogen Energy, } \\
33 \text { (24), p.7479- } \\
7488, \text { Dec } 2008\end{array}$ & $\begin{array}{l}\text { Department of Chemical Engineering New } \\
\text { Mexico State University, P.O. Box 30001, } \\
\text { MSC 3805, Las Cruces, NM 88003, USA }\end{array}$ \\
\hline $\begin{array}{l}\text { Fabrication of MOF-5 } \\
\text { membranes using } \\
\text { microwave-induced rapid } \\
\text { seeding and solvothermal } \\
\text { secondary growth }\end{array}$ & $\begin{array}{l}\text { Yoo, Y. / Lai, } \\
\text { Z. / Jeong, } \\
\text { H.K. }\end{array}$ & $\begin{array}{l}\text { Microporous and } \\
\text { Mesoporous } \\
\text { Materials, } 123 \text { (1), } \\
\text { p.100-106, Jul } 2009\end{array}$ & $\begin{array}{l}\text { Artie McFerrin Department of Chemical } \\
\text { Engineering, Texas A\&M University, } \\
\text { College Station, TX 77843-3122, USA / } \\
\text { School of Chemical and Biomolecular } \\
\text { Engineering, Nanyang Technological } \\
\text { University, N1.2, 62 Nanyang Drive, } \\
\text { Singapore } 6347459 \text {, Singapore }\end{array}$ \\
\hline $\begin{array}{l}\text { High surface area } \\
\text { microporous carbon } \\
\text { materials for cryogenic } \\
\text { hydrogen storage } \\
\text { synthesized using new } \\
\text { template-based and ... }\end{array}$ & $\begin{array}{l}\text { Gregory P } \\
\text { Meisner / } \\
\text { Qingyuan Hu }\end{array}$ & $\begin{array}{l}\text { Nanotechnology, } 20 \\
\text { (20), p.204023, May } \\
2009\end{array}$ & $\begin{array}{l}\text { Materials and Processes Laboratory, } \\
\text { General Motors R\&D Center, Warren, MI } \\
\text { 48090, USA / School of Mechanical } \\
\text { Engineering, Purdue University, West } \\
\text { Lafayette, IN 47907, USA / E-mail: } \\
\text { gregory.p.meisner@gm.com }\end{array}$ \\
\hline $\begin{array}{l}\text { High Throughput } \\
\text { Microwave Synthesis of } \\
\text { Metal Organic Framework } \\
\text { (MOF) Libraries }\end{array}$ & \begin{tabular}{|l} 
Neil L. \\
Campbell, \\
Jake Grace, \\
Richard W. \\
DewsonF, \\
Jean-Noel \\
Rebilly, Darren \\
Bradshaw, Ben \\
Carter, Andrew \\
I. Cooper and \\
Mathew J. \\
Rosseinsky
\end{tabular} & Poster Presentation & $\begin{array}{l}\text { Centre for Materials Discovery, } \\
\text { Department of Chemistry, The University } \\
\text { of Liverpool, Crown Street, Liverpool, L69 } \\
\text { 7ZD, United Kingdom / Chemspeed } \\
\text { Technologies, Northampton, UK }\end{array}$ \\
\hline $\begin{array}{l}\text { High-Enthalpy Hydrogen } \\
\text { Adsorption in Cation- } \\
\text { Exchanged Variants of the } \\
\text { Microporous Metal- } \\
\text { Organic Framework Mn3 } \\
\text { [(Mn4Cl)3(BTT)8(CH3OH) } \\
10] 2\end{array}$ & $\begin{array}{l}\text { Mircea Dinca` } \\
\text { and Jeffrey R. } \\
\text { Long* }\end{array}$ & $\begin{array}{l}\text { J. AM. CHEM. SOC. } \\
9 \text { VOL. } 129, \text { NO. } 36 \text {, } \\
2007\end{array}$ & $\begin{array}{l}\text { Department of Chemistry, UniVersity of } \\
\text { California , Berkeley , California } 94720\end{array}$ \\
\hline $\begin{array}{l}\text { Hydrogen adsorption } \\
\text { equilibrium and kinetics in } \\
\text { metal-organic framework } \\
\text { (MOF-5) synthesized with } \\
\text { DEF approach }\end{array}$ & $\begin{array}{l}\text { Saha, D. / Wei, } \\
\text { Z. / Deng, S. }\end{array}$ & $\begin{array}{l}\text { Separation and } \\
\text { Purification } \\
\text { Technology, } 64 \text { (3), } \\
\text { p.280-287, Jan } 2009\end{array}$ & $\begin{array}{l}\text { Department of Chemical Engineering, New } \\
\text { Mexico State University , P.O. Box 30001, } \\
\text { MSC 3805, Las Cruces, NM 88003, USA }\end{array}$ \\
\hline $\begin{array}{l}\text { Hydrogen adsorption on } \\
\text { porous silica }\end{array}$ & $\begin{array}{l}\text { Sheppard, } \\
\text { D.A. / Buckley, } \\
\text { C.E. }\end{array}$ & $\begin{array}{l}\text { International Journal } \\
\text { of Hydrogen Energy, } \\
33 \text { (6), p.1688-1692, } \\
\text { Mar 2008 }\end{array}$ & $\begin{array}{l}\text { Department of Imaging and Applied } \\
\text { Physics, Curtin University of Technology, } \\
\text { GPO Box U 1987, Perth, WA, Australia }\end{array}$ \\
\hline $\begin{array}{l}\text { Hydrogen Storage in a } \\
\text { Microporous Metal- } \\
\text { Organic Framework with } \\
\text { Exposed Mn2+ } \\
\text { Coordination Sites [92K] }\end{array}$ & $\begin{array}{l}\text { Mircea Dinca, } \\
\text { Anne Dailly,, } \\
\text { Yun Liu, Craig } \\
\text { M. Brown, } \\
\text { Dan. A. } \\
\text { Neumann, and }\end{array}$ & $\begin{array}{l}\text { J. Am. Chem. Soc., } \\
\text { 2006, 128 (51), pp } \\
\text { 16876-16883 }\end{array}$ & $\begin{array}{l}\text { University of California , Berkeley.; General } \\
\text { Motors Corp.; Purdue University.; NIST } \\
\text { Center for Neutron Research, University } \\
\text { of Maryland; Indiana University Cyclotron } \\
\text { Facility; / jrlong@berkeley.edu }\end{array}$ \\
\hline
\end{tabular}




\begin{tabular}{|c|c|c|c|}
\hline & Jeffrey R. Long & & \\
\hline $\begin{array}{l}\text { Improved hydrogen } \\
\text { storage in the modified } \\
\text { metal-organic frameworks } \\
\text { by hydrogen spillover } \\
\text { effect }\end{array}$ & $\begin{array}{l}\text { Liu, Y.Y. I } \\
\text { Zeng, J.L. / } \\
\text { Zhang, J. / Xu, } \\
\text { F. / Sun, L.X. }\end{array}$ & $\begin{array}{l}\text { International Journal } \\
\text { of Hydrogen Energy, } \\
32(16), \text { p.4005- } \\
4010, \text { Nov } 2007\end{array}$ & $\begin{array}{l}\text { Materials and Thermochemistry } \\
\text { Laboratory, Dalian Institute of Chemical } \\
\text { Physics, Chinese Academy of Sciences, } \\
\text { Dalian } 116023 \text {, China / Graduate School of } \\
\text { Chinese Academy of Sciences, Beijing } \\
\text { 100049, China }\end{array}$ \\
\hline $\begin{array}{l}\text { Microporous metal- } \\
\text { organic framework zinc(II) } \\
\text { imidazole- } 4,5- \\
\text { dicarboxylate: Four-fold } \\
\text { helical structure and } \\
\text { strong... }\end{array}$ & $\begin{array}{l}\text { Zhong, R.Q./ } \\
\text { Zou, R.Q. IXu, } \\
\text { Q. }\end{array}$ & $\begin{array}{l}\text { Microporous and } \\
\text { Mesoporous } \\
\text { Materials, 102 (1), } \\
\text { p.122-127, May } \\
2007\end{array}$ & $\begin{array}{l}\text { National Institute of Advanced Industrial } \\
\text { Science and Technology (AIST), Ikeda, } \\
\text { Osaka 563-8577, Japan }\end{array}$ \\
\hline $\begin{array}{l}\text { Microporous Metal- } \\
\text { Organic Frameworks } \\
\text { Incorporating 1,4- } \\
\text { Benzeneditetrazolate: } \\
\text { Syntheses, Structures, } \\
\text { and Hydrogen Storage } \\
\text { Properties }\end{array}$ & $\begin{array}{l}\text { Mircea Dinca, } \\
\text { Anta F. Yu, } \\
\text { and Jeffrey R. } \\
\text { Long }\end{array}$ & & $\begin{array}{l}\text { Contribution from the Department of } \\
\text { Chemistry, University of California, } \\
\text { Berkeley, California 94720 E-mail: } \\
\text { jrlong@berkeley.edu }\end{array}$ \\
\hline $\begin{array}{l}\text { Microwave synthesis of } \\
\text { hybrid inorganic-organic } \\
\text { materials including porous } \\
\text { Cu3(BTC)2 from Cu(II)- } \\
\text { trimesate mixture }\end{array}$ & $\begin{array}{l}\text { Seo, Y.K. I } \\
\text { Hundal, G. I } \\
\text { Jang, I.T. I } \\
\text { Hwang, Y.K. I } \\
\text { Jun, C.H. / } \\
\text { Chang, J.S. }\end{array}$ & $\begin{array}{l}\text { Microporous and } \\
\text { Mesoporous } \\
\text { Materials, 119 (1), } \\
\text { p.331-337, Mar } 2009\end{array}$ & $\begin{array}{l}\text { Research Center for Nanocatalysts, Korea } \\
\text { Research Institute of Chemical Technology } \\
\text { (KRICT), P.O. Box } 107 \text {, Yusung, Daejeon } \\
305-600 \text {, Republic of Korea / Department } \\
\text { of Chemistry, Center for Bioactive } \\
\text { Molecular Hybrid, Yonsei University, } \\
\text { Seodaemoonku, Seoul 120-749, South } \\
\text { Korea }\end{array}$ \\
\hline $\begin{array}{l}\text { Ordered micro-porous } \\
\text { carbon molecular sieves } \\
\text { containing well-dispersed } \\
\text { platinum nanoparticles for } \\
\text { hydrogen storage }\end{array}$ & $\begin{array}{l}\text { Yang, Y.X. I } \\
\text { Bourgeois, L. I } \\
\text { Zhao, C. I } \\
\text { Zhao, D. I } \\
\text { Chaffee, A. I } \\
\text { Webley, P.A. }\end{array}$ & $\begin{array}{l}\text { Microporous and } \\
\text { Mesoporous } \\
\text { Materials, } 119 \text { (1), } \\
\text { p.39-46, Mar } 2009\end{array}$ & $\begin{array}{l}\text { Department of Chemical Engineering, } \\
\text { Monash University, Wellington Road, } \\
\text { Clayton, VIC } 3800 \text {, Australia / Monash } \\
\text { Centre for Electron Microscopy, Monash } \\
\text { University, VIC } 3800 \text {, Australia / } \\
\text { Department of Chemistry and Laboratory } \\
\text { of Advanced Materials, Fudan University, } \\
\text { Shanghai } 20043 \text {, PR China / State Key } \\
\text { Laboratory of Advanced Technology for } \\
\text { Materials Synthesis and Processing, } \\
\text { School of Materials Science and } \\
\text { Engineering, Wuhan University of } \\
\text { Technology, Wuhan 430070, PR China / } \\
\text { eDepartment of Chemistry, Monash } \\
\text { University, VIC 3800, Australia }\end{array}$ \\
\hline $\begin{array}{l}\text { Preparation, crystal } \\
\text { structure and properties of } \\
\text { a novel microporous Cull } \\
\text { coordination polymer } \\
\text { with... }\end{array}$ & $\begin{array}{l}\text { Du, M. / Zou, } \\
\text { R.Q. / Zhong, } \\
\text { R.Q. I } \\
\text { Yamada, T. / } \\
\text { Maruta, G. I } \\
\text { Takeda, S. / } \\
\text { Xu, Q. }\end{array}$ & $\begin{array}{l}\text { Inorganica Chimica } \\
\text { Acta, } 361 \text { (7), } \\
\text { p.1827-1831, May } \\
2008\end{array}$ & $\begin{array}{l}\text { Graduate School of Engineering, Kobe } \\
\text { University, Nada Ku, Kobe, Hyogo } 657- \\
8501 \text {, Japan / Department of Chemistry, } \\
\text { Faculty of Science and Graduate School of } \\
\text { Science, Hokkaido University, Sapporo, } \\
\text { Japan }\end{array}$ \\
\hline $\begin{array}{l}\text { Studies on MCM }-41 \\
\text { mesoporous silica for } \\
\text { drug delivery: Effect of } \\
\text { particle morphology and } \\
\text { amine functionalization }\end{array}$ & $\begin{array}{l}\text { Manzano, M. I } \\
\text { Aina, V. I } \\
\text { Arean, C.O. I } \\
\text { Balas, F. I } \\
\text { Cauda, V. I } \\
\text { Colilla, M. I } \\
\text { Delgado, } \\
\text { M.R. / Vallet- } \\
\text { Regi, M. }\end{array}$ & $\begin{array}{l}\text { Chemical } \\
\text { Engineering Journal, } \\
137(1), p .30-37, \text { Mar } \\
2008\end{array}$ & $\begin{array}{l}\text { Departamento de Química Inorgánica y } \\
\text { Bioinorgánica, Facultad de Farmacia, } \\
\text { Universidad Complutense de Madrid, } \\
28040 \text { Madrid, Spain / Departamento de } \\
\text { Química, Universidad de las Islas } \\
\text { Baleares, } 07122 \text { Palma de Mallorca, Spain }\end{array}$ \\
\hline $\begin{array}{l}\text { Synthesis and properties } \\
\text { of metal organic } \\
\text { framework compounds } \\
\text { containing Eu and } \mathrm{Tb}\end{array}$ & $\begin{array}{l}\text { Y Sato / T } \\
\text { Zhu / K } \\
\text { Uematsu / T } \\
\text { Ishigaki / K } \\
\text { Toda / M Sato, } \\
1 \text { (1) }\end{array}$ & $\begin{array}{l}2009 \text { IOP Conf. Ser.: } \\
\text { Mater. Sci. Eng. } 1 \\
012023(4 p p) \\
\text { p.012023, Feb 2009, } \\
\text { doi:10.1088/1757- } \\
\text { 8981/1/1/012023 }\end{array}$ & $\begin{array}{l}\text { Graduate School of Science and } \\
\text { Technology, Niigata University, Niigata, } \\
\text { Japan ; Department of Chemistry and } \\
\text { Chemical Engineering, Niigata University, } \\
\text { Niigata, Japan ; Center for } \\
\text { Transdiscriplinary Research, Niigata } \\
\text { University, Niigata, Japan ; E-mail: } \\
\text { msato@eng.niigata-u.ac.jp }\end{array}$ \\
\hline $\begin{array}{l}\text { Synthesis, } \\
\text { characterization and } \\
\text { hydrogen adsorption in } \\
\text { mixed crystals of MOWet } \\
\text { hydrogen peroxide } \\
\text { catalytic oxidation of olive } \\
\text { oil mill wastewaters using } \\
\text { Cu-zeolite and Cu-pillared } \\
\text { clay catalysts F-5 and } \\
\text { MOF-177 }\end{array}$ & $\begin{array}{l}\text { Saha, D. I } \\
\text { Deng, S. }\end{array}$ & $\begin{array}{l}\text { International Journal } \\
\text { of Hydrogen Energy, } \\
34 \text { (6), p.2670-2678, } \\
\text { Mar } 2009\end{array}$ & $\begin{array}{l}\text { Department of Chemical Engineering, New } \\
\text { Mexico State University, P.O. Box } 30001 \text {, } \\
\text { MSC 3805, Las Cruces, NM 88003, USA }\end{array}$ \\
\hline
\end{tabular}




\begin{tabular}{|c|c|c|c|}
\hline $\begin{array}{l}\text { Wet hydrogen peroxide } \\
\text { catalytic oxidation of olive } \\
\text { oil mill wastewaters using } \\
\text { Cu-zeolite and Cu-pillared } \\
\text { clay catalysts }\end{array}$ & \begin{tabular}{||l} 
Giordano, G. I \\
Perathoner, \\
S./ Centi, G. / \\
De Rosa, S. / \\
Granato, T. / \\
Katovic, A. I \\
Siciliano, A. / \\
(...) / \\
Tripicchio, F.
\end{tabular} & $\begin{array}{l}\text { Catalysis Today, } 124 \\
\text { (3), p.240-246, Jun } \\
2007\end{array}$ & $\begin{array}{l}\text { Department of Chemical Engineering and } \\
\text { Materials, University of Calabria, via Bucci, } \\
87030 \text { Rende, Italy / Department of } \\
\text { Industrial Chemistry and Materials } \\
\text { Engineering, University of Messina, via } \\
\text { Salita Sperone, } 98166 \text { Messina, Italy / } \\
\text { Department of "Difesa del Suolo", } \\
\text { University of Calabria, via Bucci, } 87030 \\
\text { Rende, Italy / Department of Chemistry, } \\
\text { University of Calabria, via Bucci, } 87030 \\
\text { Rende, Italy }\end{array}$ \\
\hline
\end{tabular}

http://www.micromeritics.com/Library/Bibliography-of-Papers/MOF-Metal-Organic-Fram... 3/25/2010 